\title{
Article
}

\section{Swearing as a response to pain: A cross- cultural comparison of British and Japanese participants}

Robertson, O, Robinson, Sarita Jane and Stephens, R

Available at http://clok.uclan.ac.uk/19498/

Robertson, O, Robinson, Sarita Jane ORCID: 0000-0002-4237-5412 and

Stephens, $R$ (2017) Swearing as a response to pain: A cross-cultural comparison of British and Japanese participants. Scandinavian Journal of Pain, 17 (1). ISSN 1877-8860

It is advisable to refer to the publisher's version if you intend to cite from the work. http://dx.doi.org/10.1016/j.sjpain.2017.07.014

For more information about UCLan's research in this area go to http://www.uclan.ac.uk/researchgroups/ and search for < name of research Group>.

For information about Research generally at UCLan please go to http://www.uclan.ac.uk/research/

All outputs in CLoK are protected by Intellectual Property Rights law, including Copyright law. Copyright, IPR and Moral Rights for the works on this site are retained by the individual authors and/or other copyright owners. Terms and conditions for use of this material are defined in the policies page.

\section{CLoK}

Central Lancashire online Knowledge www.clok.uclan.ac.uk

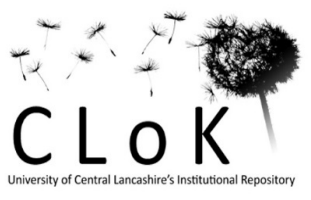


Swearing as a response to pain: a cross-cultural comparison of British and Japanese

$$
\text { participants }
$$

\author{
Olivia Robertson ${ }^{\mathrm{a} 1}$, Sarita Jane Robinson, $\mathrm{PhD}^{\mathrm{b}}$ and Richard Stephens ${ }^{\mathrm{a}}$. \\ aSchool of Psychology, University of Keele, Keele, Staffordshire, ST5 5BG. \\ bSchool of Psychology, University of Central Lancashire, Preston, Lancashire, England.
}

PR1 2HE

\title{
Number of manuscript pages: 22
}

\section{Number of Tables: 2}

1 Correspondence: Olivia Robertson. Room 1.23. School of Psychology. Faculty of Natural Sciences. Dorothy Hodgkin Building. Keele University, Keele, Staffordshire, ST5 5BG. Tel: 01782 734402. Email: O.M.Robertson@keele.ac.uk 
Background and aims: Research suggests swearing can moderate pain perception. The present study assessed whether changes in pain perception due to swearing reflect a "scripting" effect by comparing swearing as a response to pain in native English and Japanese speakers. Cognitive psychology denotes a 'script' to be a sequence of learnt behaviours expected for given situations. Japanese participants were included as they rarely, if ever, swear as a response to pain and therefore do not possess an available script for swearing in the context of pain. It was hypothesised that Japanese participants would demonstrate less tolerance and more sensitivity to pain than English participants, and - due to a lack of an available script of swearing in response to pain - that Japanese participants would not experience swearword mediated hypoalgesia.

Methods: Fifty-six native English (mean age = 23 years) and 39 Japanese $($ mean age $=21)$ speakers completed a cold-pressor task whilst repeating either a swear on control word. A 2 (culture; Japanese, British) X 2 (Word; swear; non-swear) design explored whether Japanese participants showed the same increase in pain tolerance and experienced similar levels of perceived pain when a swearing intervention was used as British participants. Pain tolerance was assessed by the number of seconds participants could endure of cold-pressor exposure and self-report pain measurements. Levels of perceived pain were assessed using a 120-mm horizontal visual analogue scale anchored by descriptors in the participant's native language of "no pain” (left) and "terrible pain” (right). The participant was asked to mark a $10 \mathrm{~mm}$ vertical line to indicate overall pain intensity. The score was measured from the zero anchor to the participant's mark.

Results: Japanese participants reported higher levels of pain $(\mathrm{p}<0.005)$ and displayed lower pain tolerance than British participants $(p<0.05)$. Pain tolerance increased in swearers regardless of cultural background $(p<0.001)$ and no interaction was found between word group and culture $(\mathrm{p}=0.96)$, thereby suggesting that swearing had no differential effect related to the cultural group of the participant.

Conclusions: The results replicate previous findings that swearing increases pain tolerance and that individuals from an Asian ethnic background experience greater levels of perceived pain than those from a Caucasian ethnic background. However, these results do not support the idea of pain perception modification due to a "scripting" effect. This is 
evidenced as swearword mediated hypoalgesia occurs irrespective of participant cultural background. Rather, it is suggested that modulation of pain perception may occur through activation of descending inhibitory neural pain mechanisms.

Implications: As swearing can increase pain tolerance in both Japanese and British people, it may be suggested that swearword mediated hypoalgesia is a universal phenomenon that transcends socio-cultural learnt behaviours. Furthermore, swearing should be encouraged as an intervention to help people cope with acute painful stimuli.

Keywords: cultural differences; pain; swearing; Japanese; cold-pressor 


\section{Introduction}

Research has shown that the act of repeating a swearword can elicit an increase in pain tolerance when compared with repeating a non-swear word $(1,2,3)$. The hypoalgesic effect has been explained as being mediated by the sympathetic nervous system triggered by swearing (3).

However, an alternative explanation posits that the act of voluntarily vocalising modulates responses to pain by engaging in a pre-learnt scripted behaviour. The term

"script" in cognitive psychology was coined to denote the idea that many transactions are stereotypical to the point that they can be written down like a script (4). It may be this scripted aspect of swearing in response to pain that produces the hypoalgesic effect by distracting attention from processing the pain response (5), perhaps by evoking familiarity or positive emotions.

One way of assessing the scripted explanation of swearing in response to pain would be a cross-cultural comparison between cultures that differ in the degree of social acceptability afforded to swearing as a pain behaviour. The English language has a colourful and expansive profane vocabulary (6) and swearing as a response to pain is culturally accepted and commonplace within British culture (7). In contrast, the Japanese language is filled with subtle verbal nuances that allow for verbal denigration to occur without profane language, and has been described as a language largely devoid of swearing $(8,9)$. For example, a Japanese speaker could cause offence by using a pronoun implying that their own status is higher than the listener (10). Anecdotal accounts indicate that Japanese speakers rarely, if ever, swear as a response to pain. Rather, onomatopoeic expressions are used in response to and as an expression of pain. For example, 'Zuki-zuki' indicates a moderate to severe throbbing pain. Forty-percent of native Japanese patients reporting tension headaches expressed their headache characteristics using zuki-zuki (11). Therefore, while the average native English speaker can be thought of as having a wellrehearsed "script" for swearing in response to pain, the average native Japanese speaker would not. Comparing the efficacy of swearing in response to pain in native English and Japanese speakers would therefore shed light on the "script" theory of hypoalgesia of swearing. 
The current study recruited 95 (56 British) participants, and asked them to complete a cold-pressor pain task whilst repeating either an English or Japanese language-specific swear or neutral (control) word. Based on previous research indicating that individuals with an Asian ethnic background demonstrate less tolerance and more sensitivity to pain than Caucasians $(12,13)$, we hypothesise that native English speakers would show increased pain tolerance and reduced pain perception compared to native Japanese speakers. Further, as Japanese speakers do not commonly use swearing in response to pain, swearing should not trigger a rehearsed "script" and so should not result in a reduced pain experience. Therefore, we would expect only English speakers to show an increased pain tolerance and reduced pain experience when they swear. 


\section{Method}

\subsection{Design:}

A 2 (culture; Japanese, English) x 2 (word intervention; swearing, non-swearing) fully independent design was implemented. Pain tolerance was measured using coldpressor latency and pain perception was self-assessed using a visual analogue scale. Participants were randomly assigned to the swearing and non-swearing conditions.

\subsection{Participants:}

Ninety-five students (59 females and 36 males; age range 18-44; mean age 22.42 years) based on an a priori power calculation. The power calculation indicated that a minimum sample size of $\mathrm{N}=90$ will allow for $80 \%$ power to detect medium sized effects ( $d=0.6)(2)$ based on comparisons across the swearing and non-swearing independent groups with alpha set at 0.05 (14). However, a slightly larger number was recruited in anticipation of possible attrition. Participants were recruited via opportunity sampling from a university in the North West of England. There were 56 native-English speaking participants (30 controls and 26 swearers, mean age 23 years), and 39 native-Japanese speaking participants (20 controls, and 19 swearers, mean age 21 years). Japanese participants had been living in the UK for a maximum of five months as part of a foreign exchange program. Thus, it is unlikely that a script of swearing in response to pain had been learnt and internalised by Japanese participants. Furthermore, whilst Japanese participants did have varying levels of English language proficiency, all participants were students undertaking a year abroad program and as such would be unlikely to have English language fluency. Participants were randomly allocated into swearing and non-swearing groups. Participants were excluded from the study applying criteria identified via a confidential self-report screening questionnaire. These criteria included: (i) hypertension; (ii) heart arrhythmia; (iii) suffering from Raynaud's Disease (iv) being prone to poor peripheral circulation in the hands/feet (v) currently feeling unwell for any reason. All participants gave verbal consent and were tested in accordance with the national and local 
ethics guidelines adhering to the Declaration of Helsinki. Participants obtained no financial reward for their participation.

\subsection{Materials and Apparatus:}

\subsubsection{Cold-pressor}

Cold-pressor pain was induced through the submergence of the non-dominant hand in ice cold water. A manual set-up, controlled container with a six-litre capacity was filled with water and cooled until a water temperature of between $1^{\circ} \mathrm{C}$ and $3^{\circ} \mathrm{C}$ was obtained. Water temperature was maintained using a mixture of ice and water. The temperature was monitored via a thermometer to ensure that the water temperature remained within the required range.

\subsubsection{Pain assessment}

Pain tolerance was measured as the time in seconds that participants were able to hold their hand immersed in the ice water. Pain perception was assessed using a visual analogue scale (VAS). The VAS was presented as a 120-mm horizontal line anchored by descriptors of "no pain” (left) and "terrible pain” (right). The participant was asked to mark a $10 \mathrm{~mm}$ vertical line to indicate overall pain intensity. The score was measured from the zero anchor to the participant's mark. A millimetre scale was used to measure the participant's score with a range of possible scores from 0 to $120(15,16)$.

\subsubsection{Words}

Four words were used for the current study. For the swear word in the British condition the word 'fuck' was chosen in order to best replicate previous studies $(1,2)$ investigating swearing as a pain response. The neutral word in the British condition was 'cup'. 'Cup' was used due to its similar linguistic qualities to the word 'fuck', as both words are one syllable long and contain hard phonemes. 
In the Japanese group the swearword 'kuso' was used. 'Kuso', which is defined as “crude for faeces; shit” by the Kodansha's Furigana Dictionary (17), was chosen because of its popularity and accessibility in contemporary Japanese. There is no equivalent to the English word 'fuck' in the Japanese language. In Japanese, the neutral word was 'kappu', which is defined as a "cup with a handle for drinks" by the Kodansha's Furigana Dictionary (17). The word 'kappu' was selected as it had similar linguistic qualities to the word 'Kuso'; both words have two syllables, and have similar starting phonemes.

\subsection{Randomisation}

Prior to the onset of data collection, a computer-generated list of random numbers was used to randomly assign participants into either the experimental or control groups, and was stratified with a 1:1 allocation using random block sizes of 2 and 3. The allocation sequence was concealed from the researchers collecting data, and was available only within sequentially numbered, but otherwise identical, sealed and opaque envelopes containing a single A4 sheet of paper with a written code designating the participant into either the intervention or control group. There were no detectable differences in weight or size between the intervention and control envelopes. Participants were blind to the alternative experimental groups, and study hypotheses were concealed until after completion of data collection. As the VAS and timed pain latency scores are not subjective measures, outcome assessors were not blinded.

\subsection{Procedure}

Participants were tested individually in a laboratory setting and a single-blind experimental design was employed. Following randomisation into an experimental group, participants completed a screening questionnaire before receiving a full briefing regarding the study. The full briefing included instructions for the cold-pressor task, information about timing and how to complete the pain scales, assertation of self-reported dominant hand, and verbal assignment of test word (control or swear) in the participant's native language. British and Japanese participants were then asked to submerge their non- 
dominant, unclenched hand into the cold water for as long as possible whilst repeating the culturally appropriate (British or Japanese) neutral or swear word. The researcher timed how long participants kept their hand submerged in the cold water, with timing starting when the participant's hand was fully immersed in the water, and stopping once the hand was fully removed. This interval was recorded in seconds as the variable pain tolerance. An upper time limit of 180 seconds was imposed. Participants were blind to the ceiling time limit to avoid participants purposefully submerging their hand until asked to stop; a behaviour which may be mediated by a range of psychosocial factors (e.g. performance bias) (18). After participants removed their hand from the water they were offered paper towels to dry their hand before completing the VAS. 


\section{Results}

The pain tolerance (time that the participant's hand remained in the iced water) and self-reported pain perception (score on the Visual Analogue Scale) were recorded and these data were then analysed using SPSS version 24. A series of 2x2 fully independent analyses of variances was used to explore the impact of culture and the intervention of swearing on pain tolerance and pain perception. Descriptive statistics were computed and normality of each variable was assessed by means of the Shapiro-Wilks test. Distributions for pain perception scores were normally distributed. The distributions for pain tolerance, however, were found to be not normally distributed. A logarithmic transformation was used to test group difference in pain tolerance, however the significance of the logarithmic transformation yielded identical results to that of the original analyses. As such the untransformed and parametric results were retained. Due to opportunity sampling procedure, $35 \%$ of the sample were male $(n=33)$, and $8.55 \%$ of participants were male Japanese $(n=8)$. As a consequence of the small group size, therefore, the genders are not covaried in the analysis.

\subsection{Pain tolerance}

Pain tolerance was recorded for participants in the swearing and non-swearing group for both the Japanese and British cultural background participants. Below Table 1 shows means and standard deviations for pain tolerance (cold-pressor latency) for the two participant groups (British and Japanese) in the swearing and non-swearing groups.

Place Table 1 around here

The ANOVA analysis revealed a significant main effect for swearing, $\mathrm{F}(1,91)=11.99, \mathrm{p}<0.001, \eta^{2}=0.12$. A comparison of the means in Table 1 indicates that swearers were able to hold their hands in the ice water for longer than the non-swearers (95\% CIs [54.66, 79.76] and [24.12, 48.87] respectively). A significant main effect of 
cultural group was also observed, $\mathrm{F}(1,91)=6.50, \mathrm{p}<0.05, \eta^{2}=0.07$. Reference to Table 1 indicates that British participants were able to tolerate the cold-pressor task for longer than their Japanese counterparts (95\% CIs [27.02, 54.05] and [51.86, 74.48] respectively). No interaction was found between word group and culture, $F(1,91)=0.003, p=0.96, \eta^{2}<0.001$, suggesting swearing had no differential effect related to the cultural group of the participant.

\subsection{Pain perception}

Visual Analogue Scale (VAS) scores were recorded for participants in the swearing and non-swearing conditions for both the Japanese and British cultural background participants after cold-pressor exposure. Below Table 2 shows means and standard deviations for VAS scores for the two participant groups in both the swearing and nonswearing groups.

Place Table 2 around here

The ANOVA analysis revealed no significant main effect of swearing, $\mathrm{F}(1,91)=1.24, \mathrm{p}=0.27, \eta^{2}=0.01$, suggesting that swearing did not reduce participants' subjective reports of pain (95\% CIs [60.96, 78.01] for swearing and [54.04, 71.33] for control groups). However, a significant main effect of cultural group was found, $\mathrm{F}(1,91)=9.82, \mathrm{p}<0.005, \eta^{2}=0.10$. A comparison of the means in Table 2 indicates that British participants report lower levels of pain on the VAS than their Japanese counterparts (95\% CIs [48.73, 64.30] and [66.35, 84.97] respectively). No interaction effect was found between culture and word group, $\mathrm{F}(1,91)=0.05, \mathrm{p}=0.82, \eta^{2}=0.01$, suggesting that swearing did not impact differently on participants from these different cultural backgrounds. 


\section{Discussion}

The current research replicated previous studies finding that swearing during exposure to a cold-pressor pain stimulus increases pain tolerance $(1,2)$. In addition, the present work explored whether differences in the effectiveness of swearing as a method of pain control would be governed by social and cultural factors, namely that of a "scripting effect”. The results indicate that swearing increased pain tolerance irrespective of cultural background. Swearing as a response to pain did not differentially affect either cultural group. However, participants from a British socio-cultural background displayed a higher pain tolerance than Japanese participants. Finally, although increases in pain tolerance as a result of swearing were found on our objective measure (the time that participants held their hand in the cold water) no differences were found in subjective reporting of participants' level of pain when swearing or not swearing. Within the results there are wide range of values in standard deviations for cold-pressor latency in all experimental groups. Within cold-pressor literature, however, there is variability in the data and this is not an unexpected result $(1,2)$. The results of the current study found that swearing impacted upon pain tolerance, but not subjective self-reported levels of pain on the VAS. Participants removed their hand from the cold-pressor upon reaching their maximum pain threshold, and were then asked to rate their overall pain on the VAS. It is likely, therefore, that participants all reported levels of maximum overall pain on the VAS and as such this would explain why self-reported levels of pain were not affected by swearing. These results replicate the findings of previous studies (2) and were not unanticipated.

The observed cultural difference in pain tolerance and perception between British and Japanese participants echoes a wealth of previous research that has shown differences between ethnic groups and pain perceptions (19, 20, 21, 22, 13, 23, 24). The results support previous research which indicates that individuals from an Asian ethnic origin are more sensitive to acute experimental pain as British participants were able to tolerate pain longer, and reported lower levels of pain than their Japanese counterparts $(25,26)$.

The observed hypoalgesic effect of swearing regardless of cultural background (British or Japanese) does not support the "scripted" explanation for the efficacy of swearing as a means of pain management. Individuals from both Japanese and British cultures were more tolerant of the painful stimulus when swearing. This was not expected 
as Japanese people are not accustomed to swearing in a pain context and swearing would not cue the scripts which could induce a hypoalgesic effects. Previous research suggests that the act of vocalisation may have a hypoalgesic effect in experimental pain conditions (27). However, it is unlikely that swearing could moderate pain in the same way as saying “ow” by causing muscle movements known to reduce pain (27). This is because in the current study both the swearwords and the alternative words are likely to have induced the same muscle movements. Rather, as swearword production is associated with greater autonomic arousal than non-swearword production (28), the hypoalgesic effect is posited to occur through swearword activated mediation of the sympathetic nervous system $(1,2$, 3).

Swearing has previously been described as a form of anger expression (2). Research investigating the role of anger expression styles and pain indicates that individuals who tend to routinely express feelings of anger, such as through verbal aggression (known as "high trait anger-out), are more sensitive to both acute and chronic pain (29). Contrariwise, however, high trait anger-out individuals have been found to experience an increase in pain tolerance when allowed to express anger in response to pain (29) - a phenomenon known as the matching hypothesis (30). Thus, it may be suggested that participants in swearword conditions experience hypoalgesia due to the opportunity for anger expression by means of swearing. Unpublished data (31) investigating the role of trait-anger and levels of pain tolerance and perception when swearing in a cold-pressor paradigm, however, indicates that levels of trait-anger - as measured by the Spielberger Anger Expression Inventory Questionnaire (32) - did not predict pain tolerance, pain perception, or heart rate. It may be a possibility, therefore, that swearword related hypoalgesia is not associated with anger expression, but may instead be involved with heightened emotionality.

Swearing is an emotional linguistic process and can induce heightened emotionality (33). Moreover, there is evidence indicating that heightened emotionality, even in complex emotional states which incorporate cognitive and emotional factors in a fashion similar to swearing, can affect the subjective experience of pain - even when pain is acute (34). Correspondingly, within psychosocial pain interventions, emotional distraction techniques have been shown to reduce levels of, and increase tolerance to pain $(35,36)$. Attentional and emotional modulation of pain is thought to occur through activation of descending 
inhibitory neural mechanisms which impact upon spinal nociceptive processes (5). The results of the current study indicate that although Japanese participants had a lower pain threshold, the intervention of swearing was effective in increasing pain tolerance. This effect was present in participants who swore irrespective of their cultural background. Thus, swearing as an emotional linguistic process could have induced activation of descending modulatory neural pain pathways; thereby altering the pain experience (37).

Whilst transient experimental pain does not replicate the complex and nuanced sensory and affective facets of clinical pain, the results of the current study may have implications for future understandings of the pain experience. Future research could further explore how other forms of voluntary vocalisations in response to pain - such as saying “ow" - affect the pain experience cross-culturally, and whether "scripting” explains the hypoalgesia associated with other habitual vocalisations associated with the pain experience. The more research and information available to both medical practitioners and lay-individuals on the subject of pain will lead to an increase in understanding and expectations about the complexities of the pain experience and how to cope with it. Furthermore, the current study does not investigate the effect of swearing frequency upon hypoalgesia. As such future research could investigate the role of habituation to swearing impacts upon the hypoalgesic effect found in the current study.

A possible limitation of the current study may be that of disparities in gender participation rates between the cultural groups. Previous research has found that females are more sensitive to cold-pressor pain than males (38). Within the present sample, $72 \%$ of Japanese participants were female, compared to British participants who had 55\% female participation rate. It could be argued that the difference in cold-pressor latencies between British and Japanese participants may be due to differences in gender participations rates. The shorter cold-pressor latency scores in Japanese participants is, however, not unexpected. In general, studies have found Caucasians demonstrate more tolerance and less sensitivity to pain than Asians $(12,25,13,23)$. Furthermore, planned comparisons in 
previous research indicates that the hypoalgesia associated with swearing benefits males and females similarly $(1,2)$. Thus, it is reasonable to suggest that gender distribution within the current study is not likely to have influenced the hypoalgesic effects of swearing.

A further limitation of the methodology was that the water in the cold-pressor task was uncirculated. This may have led to a reduction in the effectiveness of the pain stimulus, but it is reasonable to suggest that this will have applied equally across all participants. Therefore, it may be assumed that the internal validity of the study should not have been affected.

\section{Conclusions}

In conclusion, the present study indicates that, in line with Stephens' and colleagues research $(1,2,3)$ swearing can modify the length of time that people can tolerate a painful stimulus. However, the current study is the first to show that this increase in pain tolerance as a result of a swearing intervention can occur beyond English speaking cultures, specifically in this study within Japanese culture. It is interesting that there was no evidence of any differential effect of swearing on pain response between British and Japanese participants despite the marked differences in the connotations of swearing between the two cultures $(8,9)$; specifically, because within the Japanese language and culture, swearwords are not habitually employed as a response to pain in a similar way as in British English. These findings suggest that the hypoalgesia, which occurs consequent to swearword production, may be a universal, rather than socio-cultural, phenomenon.

\section{Implications}

This article presents evidence for an hypoalgesic effect of swearing across cultures. The observed hypoalgesic effect of swearing in native Japanese speakers indicates that the underlying mechanism is unlikely to be a scripting effect. Rather the results suggest that swearword-associated hypoalgesia is a universal phenomenon. 


\section{Ethical issues}

For the current study informed consent was required. All participants gave verbal consent and were tested in accordance with the national and local ethics guidelines adhering to the Declaration of Helsinki. Study protocol was not pre-registered.

Declaration of conflict of interests:

The authors declare that there is no conflict of interest.

This research did not receive any specific grant from funding agencies in the public, commercial, or not-for-profit sectors. 


\section{References}

1. Stephens, R., Atkins, J., \& Kingston, A. (2009). Swearing as a Response to Pain. Neuroreport, 20(12), 1056-1060.

2. Stephens, R., \& Umland, C. (2011). Swearing as a Response to Pain - Effect of Daily Swearing Frequency. The Journal of Pain, 12 (12), 1274-1281.

3. Stephens, R., \& Allsop, C. (2012). Effect of Manipulated State Aggression on Pain Tolerance. Psychological Reports, 111, 311-321.

4. Schank, R. C. (1989). An early work in cognitive science. Current Contents, 38, 14.

5. Roy, M., Lebuis, A., Peretz, I., \& Rainville, P. (2011). The modulation of pain by attention and emotion: a dissociation of perceptual and spinal nociceptive processes. European Journal of Pain, 15(6), 641-e1.

6. Pinker, S. (2007). The Stuff of Thought: Language as a Window into Human Nature. New York: Penguin Group.

7. Hunt, A., Carter, B., Abbott, J., Parker, A., \& Spinty, S. (2016). Pain experience, expression and coping in boys and young men with Duchenne Muscular Dystrophy-A pilot study using mixed methods. European journal of paediatric neurology, 20(4), 630-638.

8. Antoni, K. (2012). Kotodama and the Kojiki: The Japanese "Word Soul” between Mythology, Spiritual Magic, and Political Ideology.

9. Kosugi, H. (2010). Performative Power of Language: Japanese and Swearing. Jurnal Teknosastik, 8(2), 30-37.

10. Kindaichi, H. (2013). The Japanese Language. Tokyo: Tuttle Publishing

11. Takeshima, T., Ishizaki, K., Fukuhara, Y., Ijiri, M., Kusumi, M., Wakutani, Y., Mori, M., Kawashima, M., Kowa, H., Adachi, Y., Urakami, K., \& Nakashima, K. (2004). Population-Based Door-to-door Survey of Migraine in Japan: The Daisen Study. Headache: The Journal of Head and Face Pain, 44 (1), 8-19. 
12. Hobara, M. (2005). Beliefs about Appropriate Pain Behaviour: Cross-cultural and sex differences between Japanese and Euro-Americans. European Journal of Pain, 9 (4), 389-393.

13. Komiyama, O., Kawara, M., \& DeLaat, A. (2007). Ethnic Differences Regarding Tactile and Pain Thresholds in the Trigeminal Region. The Journal of Pain, 8 (4), 363-369.

14. Cohen, J. (1988). Statistical power analysis for the behavioral sciences (2nd ed.). Hillsdale, NJ: Lawrence Erlbaum Associates.

15. Price, D. D., McGrath, P. A., Rafii, A., \& Buckingham, B. (1983). The validation of visual analogue scales as ratio scale measures for chronic and experimental pain. Pain, 17(1), 45-56.

16. Seymour, R. A., Simpson, J. M., Charlton, J. E., \& Phillips, M. E. (1985). An evaluation of length and end-phrase of visual analogue scales in dental pain. Pain, 21(2), 177-185.

17. Kodansha’s Furigana Japanese Dictionary (1999) Kodansha International: Tokyo 18. von Baeyer, C. L., Piira, T., Chambers, C. T., Trapanotto, M., \& Zeltzer, L. K. (2005). Guidelines for the cold pressor task as an experimental pain stimulus for use with children. The Journal of Pain, 6(4), 218-227.

19. Zborowski, M. (1952). Cultural Components in Responses to Pain1. Journal of social issues, 8(4), 16-30.

20. Lipton, J. A., \& Marbach, J. J. (1984). Ethnicity and the Pain Experience. Social Science \& Medicine, 19 (12), 1279-1298.

21. Edwards, C. L., Fillingim, R. B., \& Keefe, F. (2001). Race, Ethnicity and Pain. Pain, 94 (2), 133-137.

22. Green, C. R., Anderson, K. O., Baker, T. A., Campbell, L. C., Decker, S., Fillingim, R. B., Kaloukalani, D. A., Lasch, K. E., Myers, C., Tait, R. C., Todd, K. H., \& Vallerand, A. H. (2003). The Unequal Burder of Pain: Confronting Racial and Ethnic Disparities in Pain. Pain Medicine, 4 (3), 277-294. 
23. Hsieh, A. Y., Tripp, D. A., Ji, L., \& Sullivan, M. J. L. (2010). Comparisons of Catastrophizing, Pain Attitudes, and Cold-Pressor Pain Experience between Chinese and European Canadian Young Adults. The Journal of Pain, 11, 11871194.

24. Fabian, L. A., McGuire, L., Goodin, B. R., \& Edwards, R. R. (2011). Ethnicity, Catastrophizing, and Qualities of the Pain Experience. Pain Medicine, 12 (2), 314321.

25. Watson, P. J., Latif, K., \& Rowbotham, D. J. (2005). Ethnic differences in thermal pain responses: A comparison of South Asian and White British healthy males. Pain, 118 (1), 194-200.

26. Rahim-Williams, B., Riley, J. L.,Williams, A. K. K., \& Fillingim, R. B. (2012). A Quantitative Review of Ethnic Group Differences in Experimental Pain Response: Do Biology, Psychology, and Culture Matter? Pain Medicine, 13 (4), 522-540.

27. Swee, G., \& Schirmer, A. (2015). On the importance of being vocal: saying "Ow” improves pain tolerance. The Journal of Pain, 16(4), 326-334.

28. Harris, C. L., Aycicegi, A., \& Gleason, J. B. (2003). Taboo words and reprimands elicit greater autonomic reactivity in a first language than in a second language. Applied Psycholinguistics, 24 (04), 561-579.

29. Bruehl, S., Burns, J. W., Chung, O. Y., \& Chont, M. (2009). Pain-related effects of trait anger expression: neural substrates and the role of endogenous opioid mechanisms. Neuroscience \& Biobehavioral Reviews, 33(3), 475-491.

30. Engebretson, T. O., Matthews, K. A., \& Scheier, M. F. (1989). Relations between anger expression and cardiovascular reactivity: Reconciling inconsistent findings through a matching hypothesis. Journal of Personality and Social Psychology, 57(3), 513.

31. Mann, J. (2012). Swearing as a Response to Pain - Effect of Trait Anger Type. Unpublished Manuscript.

32. Spielberger, C. D. (1999). State

-Trait anger expres Sons, Inc. 
33. Constant, N., Davis, C., Potts, C., \& Schwarz, F. (2009). The pragmatics of expressive content: Evidence from large corpora. Sprache und Datenverarbeitung, 33(1-2), 5-21.

34. Bushnell, M. C., Čeko, M., \& Low, L. A. (2013). Cognitive and emotional control of pain and its disruption in chronic pain. Nature Reviews Neuroscience, 14(7), 502-511.

35. Keefe, F. J., Porter, L., Somers, T., Shelby, R., \& Wren, A. V. (2013). Psychosocial interventions for managing pain in older adults: outcomes and clinical implications. British journal of anaesthesia, 111(1), 89-94.

36. Liu, X., Wang, S., Chang, S., Chen, W., \& Si, M. (2013). Effect of Brief Mindfulness Intervention on Tolerance and Distress of Pain Induced by Cold Pressor Task. Stress and Health, 29(3), 199-204.

37. Kwon, M., Altin, M., Duenas, H., \& Alev, L. (2014). The role of descending inhibitory pathways on chronic pain modulation and clinical implications. Pain Practice, 14(7), 656-667.

38. Hirsch, A. T., George, S. Z., Bialowsky, J. E., \& Robinson, M. E. (2008). Fear of pain, pain catastrophizing, and acute pain perception: relative prediction and timing of assessment. Journal of Pain, 9 (9), 806-812. 
Table 1: Means (standard deviations) for duration of cold pressor task performance in seconds for the two cultural groups in the swearing and non-swearing word groups.

\begin{tabular}{|c|c|c|c|c|c|c|}
\hline \multirow[b]{3}{*}{ Variables } & \multicolumn{6}{|c|}{ Cultural Group } \\
\hline & \multicolumn{2}{|c|}{ British } & \multicolumn{2}{|c|}{ Japanese } & \multicolumn{2}{|c|}{ Total } \\
\hline & \multicolumn{2}{|c|}{$N=56$} & \multicolumn{2}{|c|}{$N=39$} & \multicolumn{2}{|c|}{$N=95$} \\
\hline \multicolumn{7}{|l|}{ Cold-pressor latency } \\
\hline Swearing condition & 78.77 & $(57.22)$ & 55.65 & $(48.73)$ & 68.72 & (54.37) \\
\hline Non-swearing condition & 47.57 & $(33.85)$ & 25.42 & $(15.06)$ & 38.98 & (29.93) \\
\hline Total & 62.05 & $(48.36)$ & 40.92 & (39.11) & & \\
\hline
\end{tabular}


Table 2: Means (Standard deviations) for self-reported levels of pain on the VAS for the two cultural groups in the swearing and non-swearing word groups.

\begin{tabular}{|c|c|c|c|c|c|c|}
\hline \multirow[b]{3}{*}{ Variables } & \multicolumn{6}{|c|}{ Cultural Group } \\
\hline & \multicolumn{2}{|c|}{ British } & \multicolumn{2}{|c|}{ Japanese } & \multicolumn{2}{|c|}{ Total } \\
\hline & \multicolumn{2}{|c|}{$N=56$} & \multicolumn{2}{|c|}{$N=39$} & \multicolumn{2}{|c|}{$N=95$} \\
\hline \multicolumn{7}{|l|}{ VAS Pain Score } \\
\hline Swearing condition & 52.42 & $(27.09)$ & 72.95 & $(29.53)$ & 61.35 & (29.69) \\
\hline Non-swearing condition & 60.60 & $(28.51)$ & 78.37 & $(32.85)$ & 67.49 & (31.18) \\
\hline Total & 56.80 & $(27.91)$ & 75.59 & $(30.90)$ & & \\
\hline
\end{tabular}

